\title{
Single-photon generation with InAs quantum dots
}

\author{
Charles Santori $^{1,2,4}$, David Fattal $^{1}$, Jelena Vuckovic ${ }^{1}$, \\ Glenn S Solomon ${ }^{1}$ and Yoshihisa Yamamoto ${ }^{1,3}$ \\ ${ }^{1}$ Quantum Entanglement Project, ICORP, JST, E.L. Ginzton Laboratory, \\ Stanford University, Stanford, CA 94305, USA \\ ${ }^{2}$ Institute of Industrial Science, University of Tokyo, 4-6-1 Komaba, \\ Meguro-ku, Tokyo 153-8904, Japan \\ ${ }^{3}$ NTT Basic Research Laboratories, Atsugishi, Kanagawa, Japan \\ E-mail: chars@stanford.edu \\ New Journal of Physics 6 (2004) 89 \\ Received 3 February 2004 \\ Published 29 July 2004 \\ Online at http://www.njp.org/ \\ doi:10.1088/1367-2630/6/1/089
}

\begin{abstract}
Single-photon generation using InAs quantum dots in pillar microcavities is described. The effects on performance of the excitation wavelength and polarization, and the collection bandwidth and polarization, are studied in detail. The efficiency and photon state purity of these devices have been measured, and issues affecting these parameters are discussed. Prospects for improved devices are also discussed.
\end{abstract}

\section{Contents}

1. Introduction 2

2. Device structure and operation 2

3. Effects of excitation and collection parameters 4

4. Efficiency and photon state purity 11

5. Future improvements 14

$\begin{array}{ll}\text { Acknowledgments } & 15\end{array}$

$\begin{array}{ll}\text { References } & 15\end{array}$

${ }^{4}$ Author to whom any correspondence should be addressed. 


\section{Introduction}

An ideal single-photon source produces exactly one photon in a definite quantum state, in contrast with a 'classical' source, such as attenuated laser pulses, for which the photon number follows a Poisson distribution. Much progress has been made recently towards such devices, especially in suppressing the probability of emitting two photons in the same pulse. Large two-photon suppression has been observed using single-quantum emitters such as molecules [1,2], diamond colour centres [3, 4], atoms [5], impurities in semiconductors [6] and quantum dots [7]-[11]. Significant progress has also been made in increasing the efficiency of these devices [12, 13], and in increasing the purity of the quantum states produced [14].

Much of this work has been motivated by proposed applications in quantum information, such as quantum cryptography (for a review see [15]), quantum networking (for a review see [16]) and linear-optical quantum computation [17]. For some implementations of quantum cryptography, such as BB84, the purity of the photon states is not very important, and even the efficiency does not need to be high in cases where the source must otherwise be attenuated to avoid photon-splitting attacks [18]. However, quantum networking and linear-optical quantum computation place much higher demands on the performance of a single-photon source, including the efficiency and the purity of the photon states. In linear-optical quantum computation, the requirements on the photon source, as well as the other optical components, are quite difficult, but theoretical advances may relax some of these requirements [19].

Semiconductor quantum dots [20] have already produced promising results as singlephoton emitters. The main difficulty with quantum dots is that they interact with a solidstate environment, necessitating cryogenic operation temperatures, and yet environment-induced decoherence is still a problem. However, these difficulties are offset by advantages such as being fixed in place, large dipole moments, and the possibility of integration into monolithic optical microcavity structures [21]-[23]. Using quantum dots embedded in pillar microcavities, we have obtained two-photon suppression factors as large as 40 [11], improved efficiencies [13] and photon state purities such that the mean wavepacket overlap between consecutive photons is as high as 0.8 [14]. The present study explains some of the details of operation of these devices, including examples of how the performance depends on the excitation and collection parameters. We also discuss some limitations of the current approach, based on rapid incoherent excitation followed by spontaneous emission, and possibilities for improved devices.

\section{Device structure and operation}

The structure of the most recent devices for which we have completed measurements is shown schematically in figure 1(a). One or more InAs quantum dots, surrounded by a GaAs matrix, are embedded in a micropillar optical cavity. The quantum dots serve as the single-photon emitters. The optical microcavity serves to modify the spontaneous emission properties of the quantum dot through the Purcell effect [24]. When a radiative transition of the quantum dot is on resonance with a cavity mode, if the quantum dot couples much more strongly with this mode than to the background 'leaky' modes, the spontaneous emission rate can increase substantially and light is emitted mainly into the cavity mode.

The quantum dots and a planar microcavity were grown together by molecular-beam epitaxy (MBE). The quantum-dot layer, formed through self-assembly, contains dots with a 


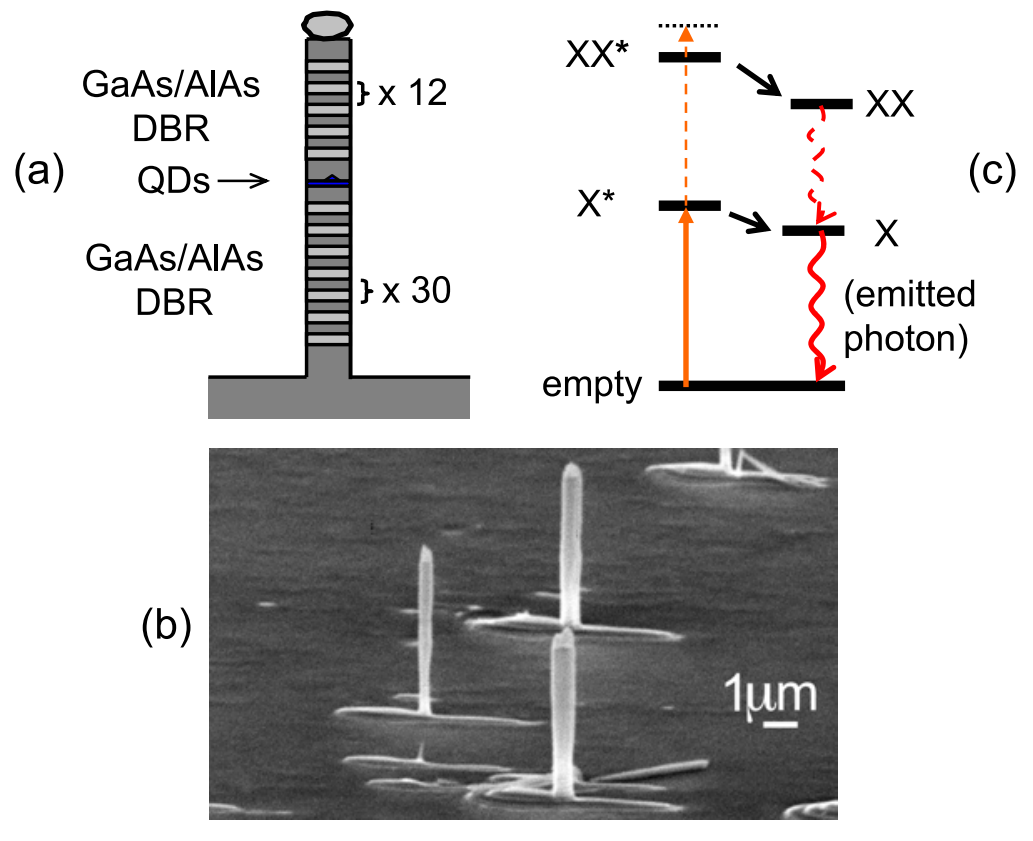

Figure 1. (a) Schematic diagram of single-photon device, (b) scanning-electron microscope image of actual pillar structures; and (c) optical excitation scheme.

density of approximately $25 \mu \mathrm{m}^{-2}$. The quantum dots are centred within a GaAs spacer layer one optical wavelength thick $(274 \mathrm{~nm})$, which is sandwiched between two distributed-Braggreflector (DBR) mirrors. The DBR mirrors consist of alternating layers of GaAs and AlAs, with designed thicknesses of 68.6 and $81.4 \mathrm{~nm}$, respectively, which are both approximately one-quarter wavelength thick. The wafer was etched to form micropillars, about $5 \mu \mathrm{m}$ tall, using sapphire dust particles as etch masks. These particles were spread randomly onto the sample, and had a distribution of sizes and shapes, leading to pillars with diameters ranging from 0.3 to $>5 \mu \mathrm{m}$, as seen in figure 1(b). The straightness of the pillar sidewalls results from a particular etching process, chemically assisted ion-beam etching (CAIBE). The pillar structures served as three-dimensional optical microcavities, with quality factors as large as 1270 and mode volumes of only a few cubic wavelengths. Often, the fundamental mode was observed to have polarization non-degeneracy, due to asymmetry in the pillar cross-sections, with wavelength splittings of the order of $1 \mathrm{~nm}$. The cavities produced measured spontaneous emission rate enhancement factors as large as 5 [11]. As a result, the spontaneous-emission lifetime for quantum-dot radiative transitions on resonance with a cavity could be as short as $100-300$ ps. It is usually acceptable to have several quantum dots contained within a pillar, since the emission wavelengths of the dots vary randomly over a wide distribution due to fluctuations in size and composition.

The operation scheme is shown in figure 1(c). A short (2-3 ps) optical pulse generated by a tunable Ti-sapphire laser raises the quantum dot into an excited state containing one electronhole pair. The quantum dot then quickly relaxes (with a timescale of the order of $10 \mathrm{ps)} \mathrm{to} \mathrm{a}$ lowest excited state. This state then decays through a much slower spontaneous emission process (100-300 ps) to emit a single photon. The spontaneous emission is collected and sent through a narrow-band $(0.1 \mathrm{~nm})$ spectral filter. This not only removes background emission from the sample, but also protects against events in which the quantum dot receives multiple excitations. In these events, multiple photons are emitted, but each photon has a unique wavelength, as a 
result of the electrostatic interactions between particles inside the quantum dot, leading to energy shifts of the order of meV [25].

In the experiments described below, the sample was cooled to temperatures ranging from 3 to $10 \mathrm{~K}$ using a liquid-helium continuous-flow cryostat. In this cryostat, the sample could be positioned close to a thin window, so that high-resolution imaging was possible. Devices on the sample were excited, unless noted otherwise, from a steep angle by a laser beam. The resulting emission was collected from above with a lens ( 0.5 numerical aperture), imaged onto a pinhole to control the collection region on the sample and sent to various measuring devices.

\section{Effects of excitation and collection parameters}

The way in which the quantum dot is excited by the laser, and the way in which the emitted photons are filtered, have a large effect on the performance of a single-photon device. Here, we describe the effects of excitation wavelength and polarization, and of collection bandwidth and polarization.

We have two general methods of exciting a quantum dot optically. In above-band excitation, the laser frequency is tuned above the bandgap of the GaAs material surrounding the quantum dot. In this way, many free electrons and holes are created, some of which then relax into lowerenergy wetting layer levels, and then into localized quantum dot states. In resonant excitation, the laser frequency is tuned below the GaAs and wetting-layer continuum states, and the quantum dot is excited directly, as in figure 1(c).

The different behaviours produced by these two excitation methods are apparent in the photoluminescence spectra. In figure 2(a), a device which we designate as dot A was excited at $750 \mathrm{~nm}$, above the GaAs bandgap, whereas in figure 2(b), this device was excited resonantly at $909 \mathrm{~nm}$. Many more bright spectral lines are observed in the above-band case. One reason for this is that, with above-band excitation, electrons and holes are added to the quantum dot separately, leading to a fluctuation of the charge of the quantum dot. In this way, charged-exciton (trion) states are frequently produced, which emit at unique wavelengths. In addition, the spectral lines in figure 2(a) could originate from more than one quantum dot within the pillar. With resonant excitation, it is possible to selectively excite a single quantum dot. Another difference between the two spectra is the presence of some weak broadband emission in figure 2(a). The origin of this broadband emission is uncertain, but perhaps it is due to the influence of nearby charges on the recombination wavelength of excitons within the quantum dot. The position of this emission marks the fundamental (polarization-non-degenerate) optical cavity modes of this pillar, since emission on resonance with these modes is enhanced through the Purcell effect. This broadband emission provides a way of locating the cavity modes and determining their quality factors. In figure 2(c), the above-band excitation was used again, with the laser power greatly increased to saturate the quantum-dot emission. In this case, the shapes of the cavity modes are more easily seen, and the polarization non-degeneracy is also apparent.

The excitation parameters, as well as the collection filtering parameters, are important for eliminating two-photon events. Photon correlation data from dot A are shown in figure 3 to demonstrate this point. These data were obtained with a Hanbury Brown and Twiss-type set-up, which has become quite standard. Our set-ups are described in more detail elsewhere $[8,14,26]$. The collected emission from the quantum dot is spectrally filtered and split into two paths by a beamsplitter, each path leading to a photon counter. Coincidence-counting electronics generates 

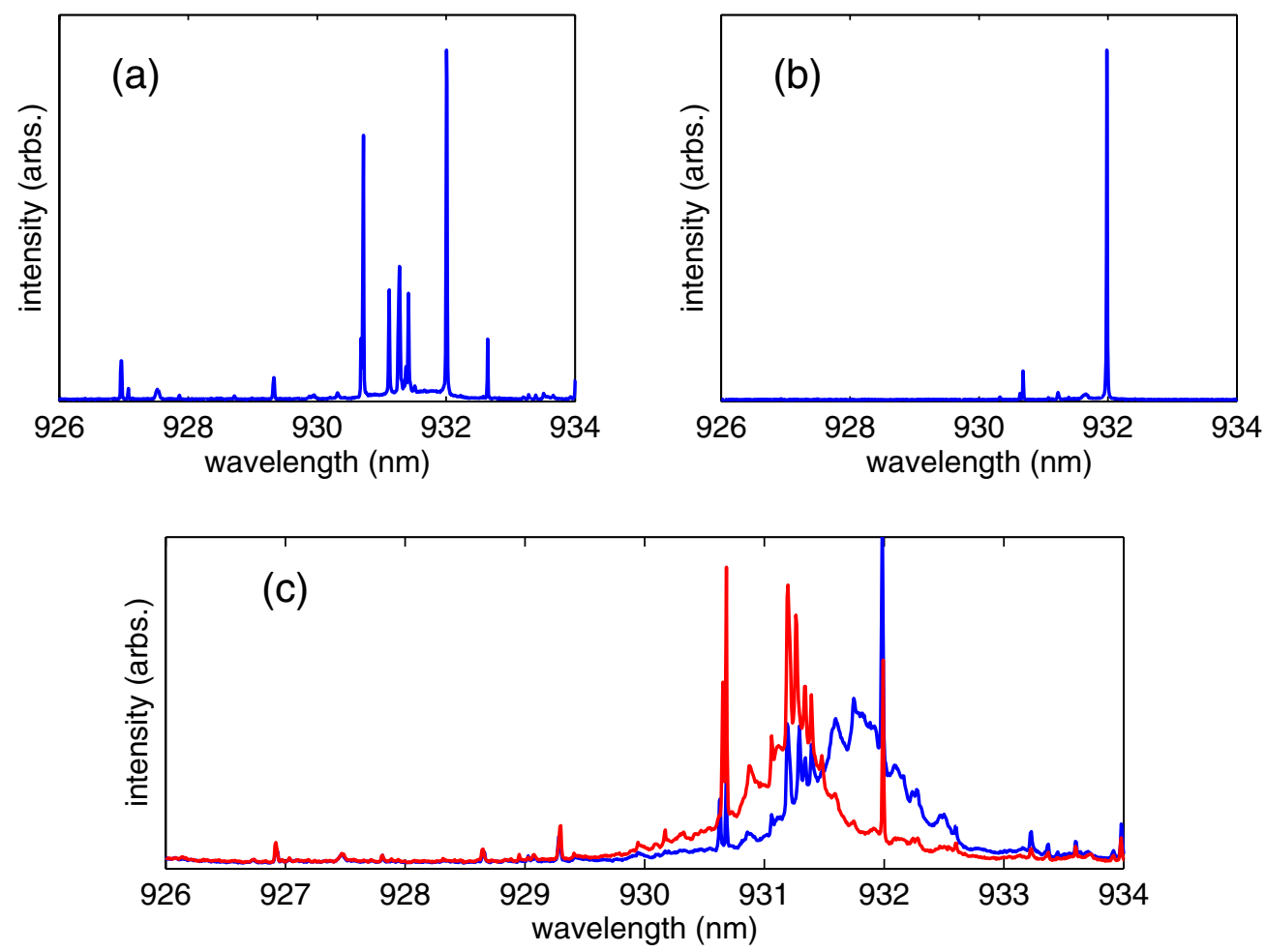

Figure 2. (a) Photoluminescence (PL) spectrum of dot A with above-band excitation at $750 \mathrm{~nm}$, (b) PL spectrum with resonant excitation at $909 \mathrm{~nm}$ and (c) saturated PL spectra under above-band excitation, showing cavity modes. Orthogonal linear polarizations were measured separately, designed $H_{A}$ (blue) and $V_{A}$ (red).

a histogram of the relative delay $\tau=t_{2}-t_{1}$ between photon detection events at counters 1 and 2 . The peak at $\tau=0$ corresponds to events in which two photons were detected in the same pulse, and thus the first goal in developing a single-photon source is to make the area of this peak as small as possible. The peaks at $\tau=n T_{\text {rep }}$, where $n$ is a non-zero integer, and $T_{\text {rep }}=13 \mathrm{~ns}$ is the laser repetition period, correspond to events in which one photon was detected from each of two different pulses. These peaks provide information about long-time-scale memory effects (blinking) in the quantum dot.

The photon correlation data in figure 3(a) were obtained from dot A under pulsed, aboveband excitation at $750 \mathrm{~nm}$. The light was collected from the bright emission line at $932 \mathrm{~nm}$ in figure 2. The spectral filtering bandwidth was about $0.4 \mathrm{~nm}$. The laser power was set in this case such that the collected count rate was less than one-half of its saturated value. The two-photon suppression relative to an equivalent Poisson source can be estimated by comparing the area of the central peak with the areas of the more distant side peaks. In this way, we estimated that the probability of emitting two photons in the same pulse was approximately $g_{0}^{(2)}=0.3$ times that for a Poisson-distributed source of the same intensity. This is unremarkable compared with other single-photon sources and, furthermore, there is some ambiguity in calculating this value due to the background floor between the peaks. These background counts originate from actual emission from the device. The calculated peak areas depend on what integration window is used. 

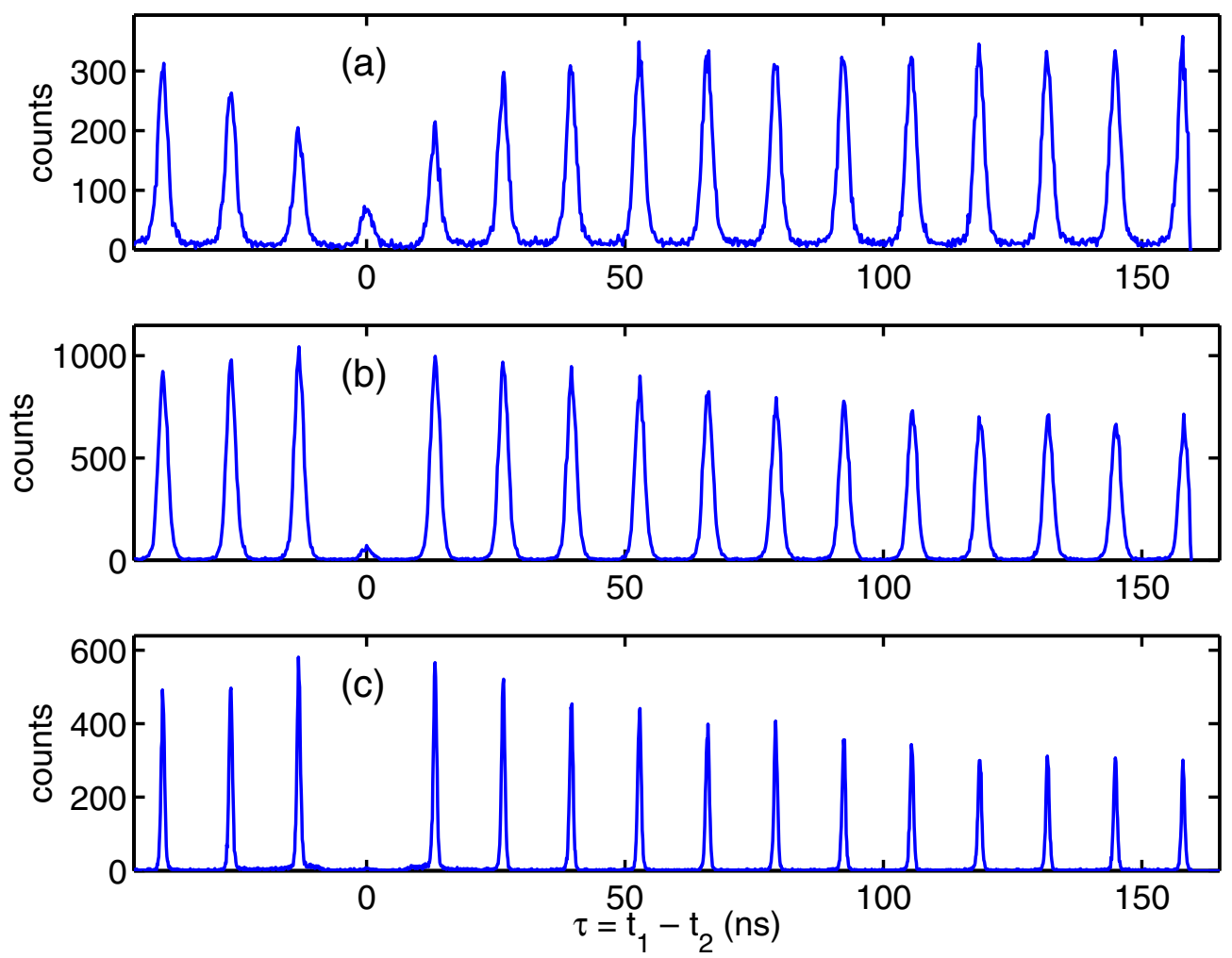

Figure 3. Photon correlation $\left(g^{(2)}\right)$ measurements with (a) above-band excitation at 750 and $0.4 \mathrm{~nm}$ collection bandwidth; (b) resonant excitation at 909 and $0.4 \mathrm{~nm}$ collection bandwidth; (c) resonant excitation at 904 and $<0.1 \mathrm{~nm}$ collection bandwidth. The narrower peaks in (c) are mainly due to the improved time resolution of the set-up used for that measurement.

This choice depends on what assumptions one is willing to make about the ability to perform time gating in an actual application.

Three problems related to the above-band excitation process are probably responsible for this poor performance. First, the process by which free electrons and holes created in the surrounding GaAs are captured into the quantum dot is more complicated than in the resonant-excitation case. The capture process can last longer, and if electrons and holes are injected randomly into the quantum dot over a time interval that is not negligible compared with the exciton recombination lifetime, it is possible for the quantum dot to capture an additional electron-hole pair after a first excitonic photon has been emitted. When this occurs, multiple photons are emitted at the single-exciton wavelength. Secondly, the broadband emission surrounding the main emission line in figure 2(a) contaminates the signal. Finally, the background floor that fills the entire region between the peaks in the photon correlation histogram suggests an additional, long-timescale component of the carrier capture process. This is observed despite the fast $(<60 \mathrm{ps})$ rise-time seen in streak-camera measurements under above-band excitation. The origin of this additional slow component is uncertain, but we typically observe it only with above-band excitation.

In figure 3(b), resonant excitation was used, again with a collection bandwidth of approximately $0.4 \mathrm{~nm}$. The performance is considerably improved. The background floor between the correlation peaks has disappeared almost entirely, and $g_{0}^{(2)}$ is now only about 0.11 . We 
attribute this improvement to the much shorter relaxation process that must occur following the excitation pulse. In figure 3(c), resonant excitation was again used, but with a narrower collected spectral bandwidth of approximately $0.1 \mathrm{~nm}$. This further improved the $g_{0}^{(2)}$ value to 0.05 . This spectral filtering was accomplished by collimating the collected light, sending it to a diffraction grating (1800 grooves $/ \mathrm{mm}$ ), and focusing the first-order diffraction into a single-mode fibre. We estimate that the coupling efficiency into the fibre was about $50 \%$.

An additional difference in the photon correlation data between the above-band and resonant excitation cases is the behaviour of the side peaks. In the above-band case, the innermost side peaks near $\tau=0$ are smaller, compared with the more distant ones. In the resonant case, the innermost side peaks are larger. In fact, if one plots the areas of these side peaks versus peak number, the behaviour is typically well described by a simple two-sided decaying exponential. We have studied this behaviour in detail in reference [26]. This behaviour most likely originates from charge fluctuation within the quantum dot. With above-band excitation, singly charged states are a necessary intermediate step in bringing the quantum dot from its empty state to a single-exciton state, containing one electron-hole pair. With resonant excitation, entire excitons are created directly within the quantum dot, and singly charged states are unwanted shelving states that can produce the observed blinking behaviour.

So far, we have only discussed the advantages of resonant excitation in terms of decreased multi-recombination events and reduced background emission. However, another possible advantage is that the exciton population statistics in the quantum dot can be made sub-Poissonian. In this case, the emitted light can be sub-Poissonian even before spectral filtering. This is a difficult issue to study with microcavity devices, since the microcavity already acts as a spectral filter, attenuating the single- and multi-exciton lines unequally [27]. However, previous measurements on a sample containing quantum dots in simple mesa structures indicate that it is possible to control the photon emission statistics to a large extent simply by varying the excitation wavelength. This other sample is described in more detail elsewhere [8, 28, 29]. For the measurements to be described next, emission was collected from a quantum dot which we designate dot $\mathrm{B}$, having a single-exciton emission wavelength of $876.1 \mathrm{~nm}$ and a bi-exciton wavelength of $877.3 \mathrm{~nm}$. The collected emission was filtered with a $2 \mathrm{~nm}$ bandwidth, centered such that both the single- and bi-exciton wavelengths were collected.

Figure 4(a) shows a photoluminescence excitation (PLE) curve for this quantum dot. The intensity of the single-exciton line was measured as the laser wavelength was varied. The arrows indicate the excitation wavelengths used for the photon correlation measurements. When the laser is tuned to the PLE resonance at $858.7 \mathrm{~nm}$, the emission intensity approximately follows $I / I_{0}=1-\mathrm{e}^{-P / P_{0}}$, where $I$ and $P$ are the collected intensity and the excitation power, respectively, and $P_{0}$ and $I_{0}$ are parameters characterizing the saturated power and intensity. For the photon correlation measurement in figure 4(b), the laser was tuned to this resonance, and the excitation power was set such that $P / P_{0} \approx 0.75$. The result shows antibunching, even though both the single- and bi-exciton lines were collected. The strength of this effect decreases as the laser power increases. When the laser is tuned slightly to lower energy, as indicated by the other arrow in figure 4(a), the opposite behaviour is observed. Figure 4(c) shows a photon correlation measurement with below-resonant excitation, and the same power as before. The large central peak indicates photon bunching, or super-Poisson statistics.

This behaviour can be explained as follows. We assume here that the laser pulse excites the quantum dot almost instantaneously compared with the exciton recombination lifetimes. The excitation then decays through a radiative cascade, with each electron-hole pair producing 

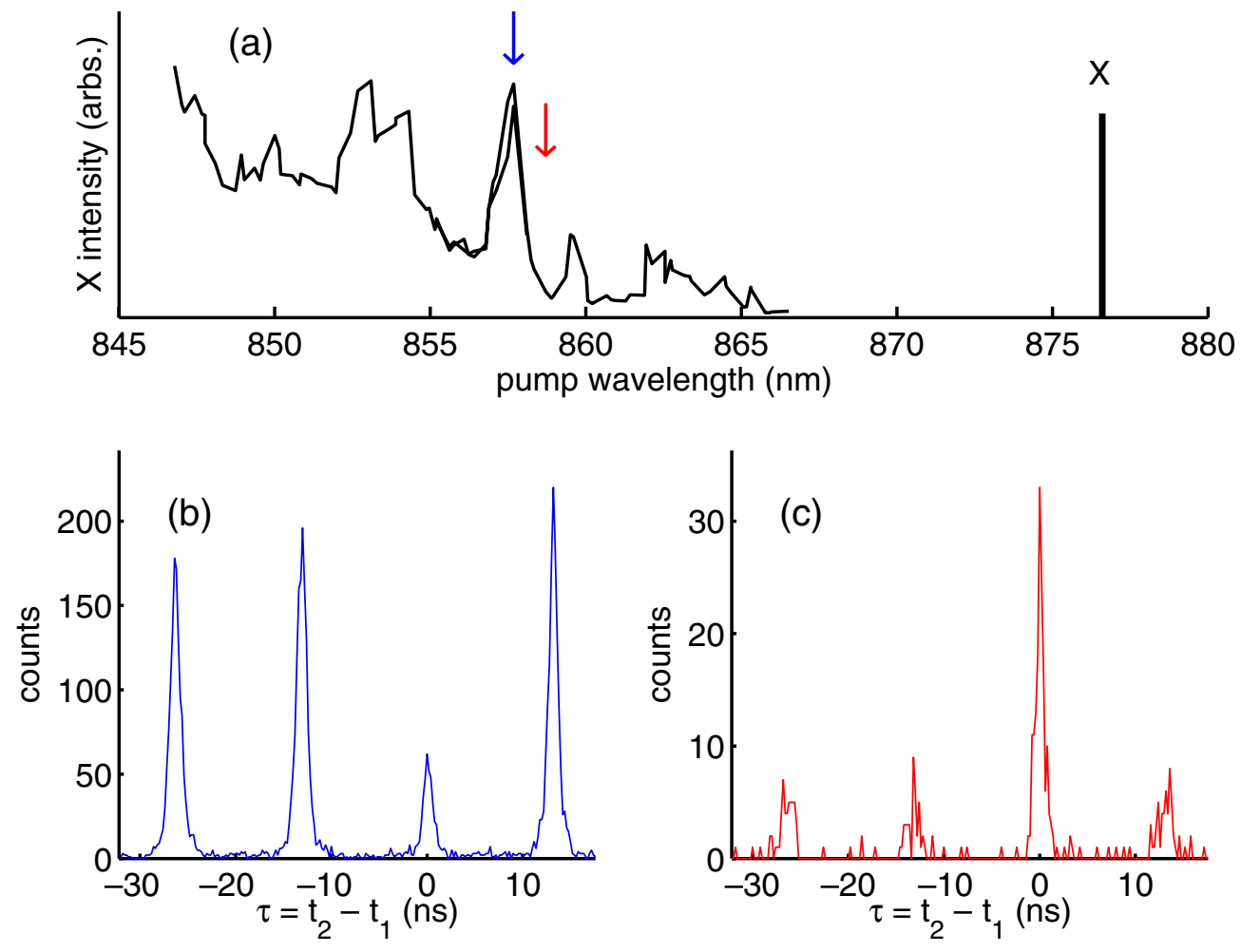

Figure 4. (a) Photoluminescence excitation (PLE) spectrum of dot B, showing intensity of single-exciton line $(X)$ versus excitation wavelength. (b) Photon correlation measurement with resonant excitation, as indicated by the blue arrow above. (c) Photon correlation measurement with below-resonant excitation, as indicated by the red arrow above.

one photon. If we consider only the single- and bi-exciton emission, it can be shown that

$$
g_{0}^{(2)}=\frac{\langle n(n-1)\rangle}{\langle n\rangle^{2}}=\frac{2 P_{2}}{\left(P_{1}+2 P_{2}\right)^{2}}=\frac{x}{\left(1+x P_{1}\right)^{2}}
$$

where $n$ is the collected photon number in a single pulse, $P_{1}$ the probability of the dot to emit just one photon, $P_{2}$ the probability to emit two photons and we define $x$ such that $P_{2}=\frac{1}{2} x P_{1}^{2}$. Note that the measured $g_{0}^{(2)}$ is independent of collection efficiency. When we primarily excite a one-photon transition, as in figure 4(b), we expect $x<1$, and thus $g_{0}^{(2)}<1$. In figure 4(c), the below-resonant laser can excite a two-photon transition, such that $2 E \approx 2 E_{X}-E_{b}$, where $E$ is the laser photon energy, $E_{X}$ the single-exciton energy and $E_{b}$ is a bi-exciton binding energy. When this two-photon transition is favoured, we can have $x>1$, and thus $g_{0}^{(2)}>1$ if $P_{1}$ is small. For this quantum dot, $E_{b}=1.9 \mathrm{meV}$ has been measured for a bi-exciton in its lowest-energy state, although in the excitation scheme we are considering, a bi-exciton would be created in an excited state, and thus $E_{b}$ is not known. The bunching effect observed here is different from the large cross-correlation that can be observed between the single- and bi-exciton lines when the probability of creating a single exciton is much smaller than one [30]. Observation of the cross-correlation effect requires that one photon counter collect only the bi-excitonic emission. 


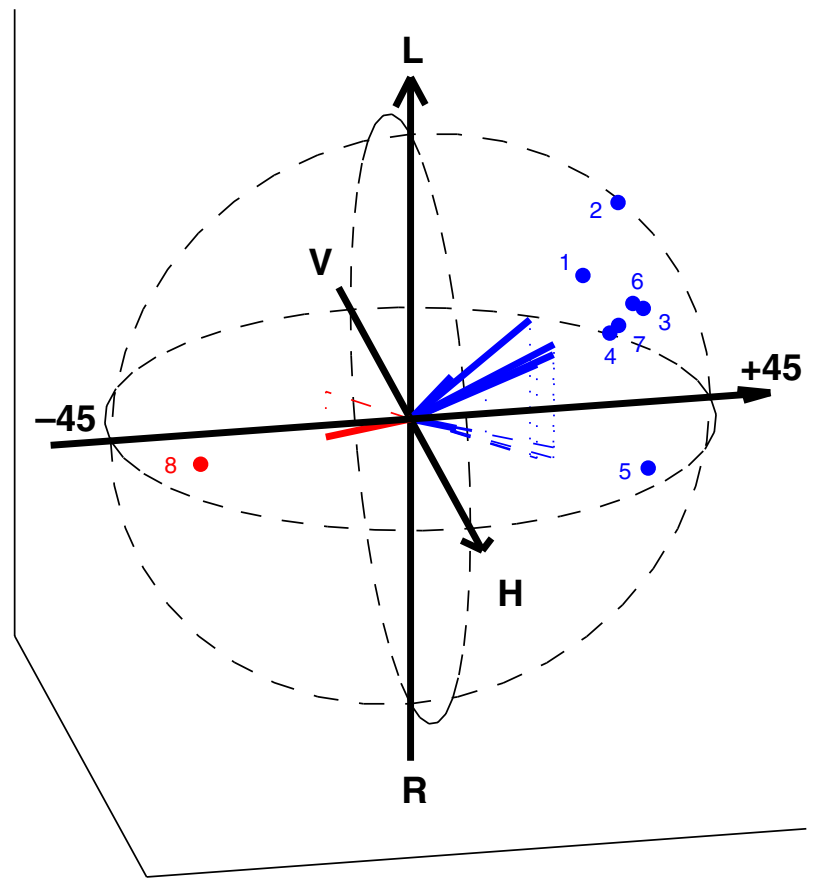

Figure 5. Emission polarizations from the single-exciton $(X)$ line of dot B with normal-incidence resonant excitation, represented on a Poincaré sphere. The excitation polarizations were (1) $H$, (2) $V$, (3) $+45^{\circ}$, (4) $-45^{\circ}$, (5) RCP, (6) LCP, (7) $\left[+\frac{1}{2},+\frac{1}{2},-\frac{1}{\sqrt{2}}\right]$ and (8) $\left[-\frac{1}{2},-\frac{1}{2},+\frac{1}{\sqrt{2}}\right]$.

In the measurement described here, both photon counters detected both the single- and bi-exciton emission.

Finally, we consider the issue of polarization, both for excitation and collection. If one can control the polarization of the emitted photons, this effectively becomes a factor of 2 improvement in efficiency compared with the random case. Furthermore, selecting the correct polarization is important for the coherence properties of a single-photon source.

It is known from PLE studies in quantum dots [31] that the laser polarization can be used to control the quantum-dot emission polarization, at least to a certain extent. This can be true even when the laser is resonant with an excited state of the quantum dot, and intraband relaxations occur before a photon is emitted. In our samples, we typically observe large polarization anisotropy, resulting from asymmetry in the quantum-dot structures. It is known that asymmetry can cause the single-exciton line to split into two components with orthogonal linear polarizations, through the electron-hole exchange interaction [32]. Thus, we can only control the polarization in a linear basis. For the pillar cavity structures, the issue is further complicated by the fact that the cavity can have a polarization splitting of the fundamental mode, as is apparent in figure 2(c). However, figure 5 shows the results of an attempt to control the emission polarization of dot $\mathrm{B}$, which does not have an optical cavity, through the excitation polarization. In these particular measurements, the quantum dot was excited from directly above the sample, through the collection lens. Eight different excitation polarizations were used, as listed in the figure caption. The last two excitation polarizations are specified by Stokes vector components in the 

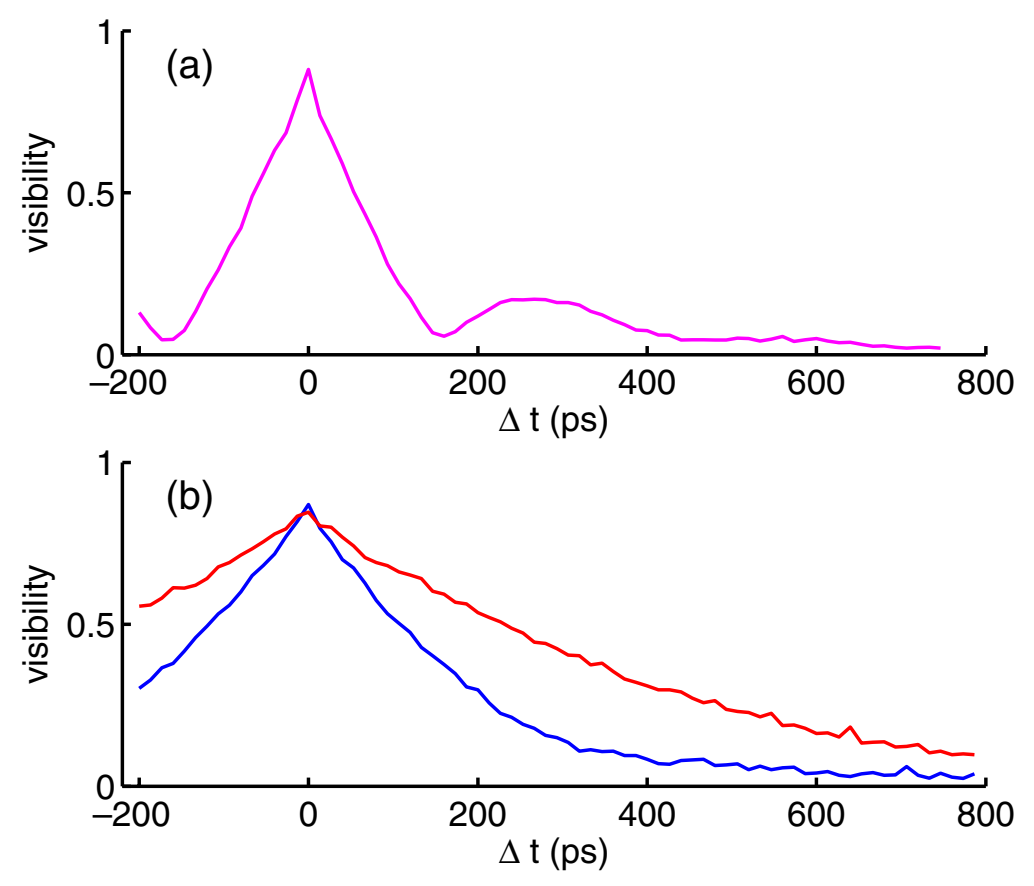

Figure 6. Measurements with a Michelson-type interferometer, showing fringe contrast versus path-length difference: (a) dot A with both polarizations detected; (b) dot A with $H_{A}$ (blue) and $V_{A}$ (red) polarizations detected separately.

order $\left[H-V, 45^{\circ}-\left(-45^{\circ}\right), R-L\right]$. The corresponding emission polarizations are plotted on a Poincaré sphere. For most excitation polarizations, the emission occurs preferentially along a mostly linear polarization, $H_{B}$. Only for a specially chosen excitation polarization, the orthogonal polarization $V_{B}$ was made to dominate. The collected $H_{B}$-polarized emission was generally stronger than the $V_{B}$ emission for this quantum dot. Anisotropy in efficiency was typical for quantum dots on this sample.

When a quantum dot with asymmetry-induced polarization splitting is placed inside an optical cavity that also has polarization splitting of its fundamental mode, interesting effects can be observed, especially if the asymmetry axes of the quantum dot and those of the cavity nearly coincide. Quantum dot A, introduced above, shows such behaviour. This quantum dot has narrow enough linewidths that the asymmetry-induced splitting can be spectrally resolved. To perform high-resolution spectroscopy, we sent the collected emission into a Michelson-type interferometer. The interference fringe contrast was recorded as a function of the difference in path lengths between the two arms of the interferometer. The result for this quantum dot, when both polarizations were collected, is shown in figure 6(a). The observed oscillations indicate a doublet with an energy splitting of about $13 \mu \mathrm{eV}$. When the individual components of this doublet, which we designate as $H_{A}$ and $V_{A}$, are collected, the oscillations disappear, as shown in figure 6(b). Instead, we observe a simple two-sided exponential decay, corresponding to a Lorentzian line shape. Interestingly, the decay constants (coherence lengths) of the two components differ.

This difference in coherence length is caused by the cavity mode splitting. As can be seen in figure 2(c), the emission line at $832 \mathrm{~nm}$ is nearly on resonance with the $H_{A}$-polarized cavity mode, but fairly far off of resonance with the $V_{A}$-polarized mode. As a result, the $H_{A}$-polarized 
exciton state in the quantum dot receives a larger Purcell enhancement than the $V_{A}$-polarized component. This was confirmed directly through polarized spontaneous emission lifetime measurements. The shorter lifetime of the $H_{A}$-polarized emission ( $\left.170 \mathrm{ps}\right)$ compared with the $V_{A}$-polarized emission $(320 \mathrm{ps})$ is reasonably consistent with the shorter coherence length.

Thus, the choice of excitation and collection polarizations can affect the behaviour of a single-photon source. For the pillar devices we tested, whenever we could resolve a polarization splitting, we selected only the brighter of the two polarizations through our collection optics. Then, we adjusted the excitation polarization to optimize the intensity of the chosen component. In this way, we achieved $g_{0}^{(2)}$ values as low as 0.02 [11].

\section{Efficiency and photon state purity}

Finding devices with small $g_{0}^{(2)}$ values on this sample was not difficult. Most devices that emitted bright spectral lines on resonance with a cavity mode could produce large two-photon suppression factors under the excitation and collection conditions described. Efficiency and photon state purity are more difficult issues, however. These depend critically on the microcavity characteristics, and on non-ideal aspects of quantum dots.

The total efficiency depends on several factors. First is the efficiency of the laser excitation. It has been shown that the quantum dot can be driven to saturation with only a moderate increase in the $g_{0}^{(2)}$ value [8]. However, the blinking effects we have observed indicate that, even at saturation, the quantum dot is not prepared deterministically in a single-exciton state. This is due to shelving states that are probably charged states [26]. This blinking effect could reduce the internal quantum efficiency by a factor of $\approx 2$. Furthermore, if the single-exciton state is not prepared with a definite polarization, the efficiency is further reduced, assuming we want just one polarization. When the correct single-exciton state is successfully prepared, the efficiency of emitting a photon into the cavity mode is approximately $\beta=\gamma_{c} /\left(\gamma_{c}+\gamma_{l}+\gamma_{n r}\right)$, where $\gamma_{l}$ is the spontaneous emission decay rate into leaky modes, and $\gamma_{n r}$ is the nonradiative decay rate (often assumed to be small in InAs quantum dots). In the best case, when the quantum dot is located at the field maximum of the cavity mode and is tuned exactly on resonance, $\gamma_{c}$ is given by [23]

$$
\frac{\gamma_{c}}{\gamma_{0}}=\frac{3 Q \lambda_{n}^{3}}{4 \pi^{2} V}
$$

Here, $Q$ is the quality factor of the cavity, $\lambda_{n}$ the effective wavelength in the semiconductor medium, $V$ the effective mode volume and $\gamma_{0}$ the radiative decay rate expected in a bulk semiconductor. We have measured quality factors as high as $Q=1270$, and the mode volume could be expected theoretically to be of the order of a few cubic wavelengths, so that we might expect $\gamma_{c} / \gamma_{0} \approx 50$ in the best cases. However, the measured increase in the decay rate when a quantum dot was on resonance with a cavity, $\left(\gamma_{c}+\gamma_{l}+\gamma_{n r}\right) /\left(\gamma_{0}+\gamma_{n r}\right)$, seldom exceeded 5. This was probably caused by a combination of factors, including random location of the quantum dot relative to the field maximum, unknown pillar diameters and random dipole orientation. Nevertheless, once a rate enhancement factor of 5 due to the microcavity resonance is observed, it is known that spontaneous emission into the cavity mode is the dominant decay path from the single-exciton state.

Finally, the efficiency depends on how much of the light that escapes from the cavity can be collected by a lens. In our set-up, we used a collection lens with a moderate numerical aperture 
of 0.5 . The best total efficiencies measured immediately after this lens for devices on the sample described here were less than $10 \%$, and efficiencies of only a few percent were common. An efficiency of $8 \%$ after the collection lens was reported in [13] for a different sample, and higher outcoupling efficiencies were inferred based on numerical simulations that predicted that the divergence angle of the light escaping from the top of the cavity exceeded the numerical aperture of the collection lens.

The purity of the photon states produced depends on at least four factors: the relaxation rate from the higher-energy single-exciton state produced by the laser to the lowest-energy exciton state, the exciton decay rate, the pure dephasing rate and polarization splitting. In the ideal case, with instantaneous initial relaxation and no pure dephasing, the time dependence of the emitted photon wavepacket in a rotating frame can be written as

$$
|\psi\rangle \propto \int_{0}^{\infty} \mathrm{d} t \mathrm{e}^{-\gamma t} a^{\dagger}(t)|0\rangle
$$

where $\gamma$ is the spontaneous-emission amplitude decay rate, and $a^{\dagger}(t)$ creates a photon at time $t$. A simple way to model a finite relaxation rate $r$ from the upper state, and also include a pure dephasing process is to replace the pure state above with a random state,

$$
|\psi\rangle \propto \int_{t_{0}}^{\infty} \mathrm{d} t \mathrm{e}^{-\gamma\left(t-t_{0}\right)+\mathrm{i} \phi(t)} a^{\dagger}(t)|0\rangle
$$

where $t_{0}$ is a random variable with probability density $r \mathrm{e}^{-r t_{0}}$ for $t_{0}>0$ and zero otherwise, and $\phi(t)$ is a random function whose probability distribution describes the pure dephasing process. For the simple case of a time-invariant pure dephasing process and a Lorentzian emission spectrum, $\left\langle\mathrm{e}^{\mathrm{i} \phi(t)} \mathrm{e}^{-\mathrm{i} \phi(t+\tau)}\right\rangle=\mathrm{e}^{-\alpha|\tau|}$, where $\alpha=1 / T_{2}^{*}$ is the rate of pure dephasing. The spectral linewidth (FWHM) in this case is $\Delta \omega=2 / \tau_{c}=2(\gamma+\alpha)$.

Polarization splitting could be an additional complication in cases where we cannot isolate just one component of a polarization-split doublet using a polarizer. This could occur, for example, if the quantum-dot asymmetry axis is misaligned with the cavity-mode splitting axis, and in this way the cavity distorts the polarization. In these cases, the collected photons will contain two distinct frequency components. The photon state can only be a pure state if these two frequency components occur as a definite linear superposition. This depends on the details of the relaxation process.

As described in more detail in [14], we have performed measurements to test the purity of the photon states produced by these devices. Results for two devices, dot A described above and another device, dot $\mathrm{C}$, are shown in figure 7. In figure 7(a), time-resolved spectroscopy using a streak camera system was used to estimate intensity decay times $\tau_{r}=1 /(2 \gamma)$ of 166 and $171 \mathrm{ps}$ for particular emission lines of dots $\mathrm{A}$ and $\mathrm{C}$, respectively. For dot A, the same line was studied as discussed previously, and the $H_{A}$-polarized component was selected. For dot $\mathrm{C}$, an extremely bright line was found at $919.5 \mathrm{~nm}$, and the quantum dot producing it was resonantly excited at $905 \mathrm{~nm}$. The emission from this pillar was already strongly polarized in one direction. In figure 7(b), measurements on the same spectral lines using the Michelson-type interferometer are shown. From these measurements, we can infer the shapes and widths of the emission lines. The spectral line from dot A has a predominantly Lorenztian shape with a $1 / e$ coherence length $\tau_{c}=223 \mathrm{ps}$. The line from dot $\mathrm{C}$ has a predominantly Gaussian shape, and the $1 / e$ coherence length is only $44 \mathrm{ps}$. A simple measure of the purity of the photon states is the ratio $2 \tau_{r} / \tau_{c}$. For 
(a)
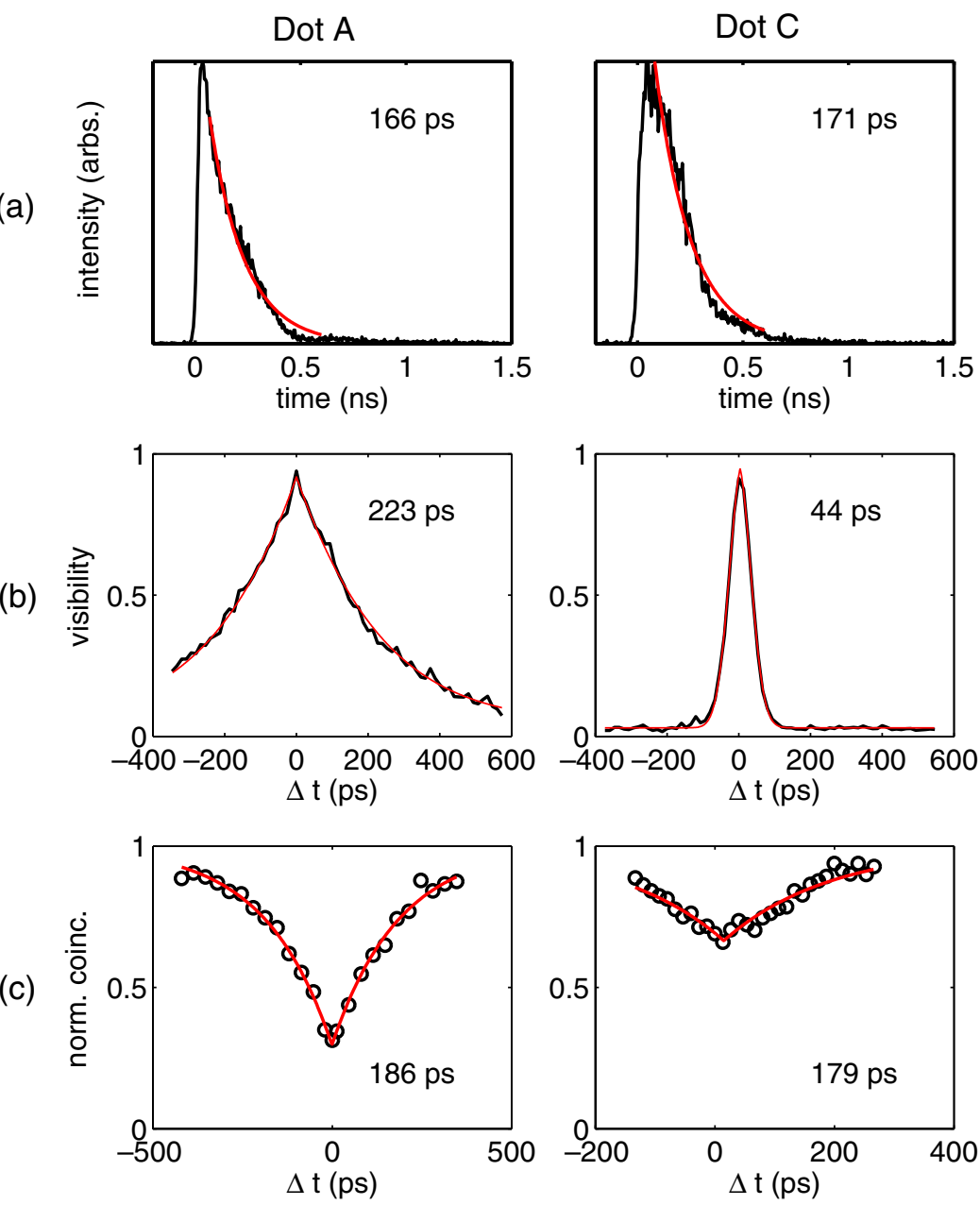

Figure 7. Measurements on dots $\mathrm{A}$ and $\mathrm{C}$ to characterize photon state purity: (a) streak camera measurements, showing mean intensity versus time following a laser pulse; (b) interferometer measurements, showing fringe contrast versus path-length difference; (c) two-photon interference measurement, showing normalized coincidence rate versus path-length difference. This normalized rate is approximately twice the probability for two photons that collide at the beamsplitter to exit in different directions.

ideal photon states as in equation (3), this ratio is equal to unity, but with the addition of pure dephasing, the ratio increases. For dot $\mathrm{A}$ this ratio is approximately 1.5 . For $\operatorname{dot} \mathrm{C}$ this ratio is less meaningful, since the spectrum is non-Lorenztian, but we obtain a value of about 8 .

Most quantum-information applications of single-photon sources require pure photon states because they employ two-photon interference in their schemes. The simplest example of twophoton interference is the Hong-Ou-Mandel experiment [33]-[35]: if two independent photons with identical wavepackets collide at a 50-50 beamsplitter, they always exit together, randomly choosing a side. They never exit in opposite directions. However, if the photons do not have identical wavepackets, they can behave independently, and the two-photon interference effect is reduced. A useful way to characterize photon state purity is to define a two-photon interference 
visibility, $V_{2}=\left(P_{\text {same }}-P_{\text {opp }}\right) /\left(P_{\text {same }}+P_{\text {opp }}\right)$, where $P_{\text {same }}$ is the probability for two photons to exit in the same direction, and $P_{o p p}$ is the probability for them to exit in opposite directions. It can be shown that $V_{2}$ is equal to the mean magnitude-squared overlap between the wavepackets of the two photons. For the photon states in equation (4), the mean overlap is

$$
V_{2}=\frac{\gamma}{\gamma+\alpha} \frac{r}{r+2 \gamma}
$$

In the limit $r \rightarrow \infty$, this is equal to the inverse of the ratio $2 \tau_{r} / \tau_{c}$ measured above.

It is possible, however, that the mean overlap between two consecutively emitted photons could be larger, if some of the spectral broadening results from a slow spectral-diffusion process. As described in [14], we tested this experimentally, by exciting the quantum dot twice by laser pulses $2 \mathrm{~ns}$ apart and arranging for the photons emitted in response to these pulses to collide sometimes at a beamsplitter. The beamsplitter outputs were measured with photon counters. The probability of two photons that meet at the beamsplitter to exit in opposite directions was estimated from measured coincidence rates. A small delay was also added which allowed the overlap between the photons to be adjusted. The results for dots $\mathrm{A}$ and $\mathrm{C}$ are shown in figure 7(c). The results indicate maximum overlaps of 0.70 and 0.33 for dots $\mathrm{A}$ and $\mathrm{C}$, respectively. These increase to 0.81 and 0.38 after correcting for known imperfections in the set-up. These values are larger than the inverses of the $2 \tau_{r} / \tau_{c}$ ratios given above. The widths of the coincidence dips, estimated by fitting a simple function of the form $1-a \exp \left(-|\Delta t| / \tau_{m}\right)$ are 186 and $179 \mathrm{ps}$ for dots $\mathrm{A}$ and $\mathrm{C}$, respectively. These are close to the measured spontaneous emission decay rates, as expected theoretically.

\section{Future improvements}

Although the present results are encouraging, the efficiency and the photon-state purity must be improved further if these devices are to be useful for many quantum-information applications. The main possibilities for improvements are to reduce the pure dephasing rate, to improve the cavity design and Purcell factors, and to modify the excitation scheme.

One of the most important questions for these devices is what is the dominant source of pure dephasing. The lack of a strong temperature dependence below $7 \mathrm{~K}$, and the wide variety of linewidths observed on this sample, suggest that for most of the devices, interaction with phonons is not the dominant pure dephasing mechanism. Another mechanism could be interaction with nearby charges [36], perhaps on pillar surfaces or in other quantum dots. In this case, experimentation with fabrication methods might yield improvements.

Both the efficiency and photon state purity could be increased by improving the optical cavities. Photonic-bandgap cavities could be used, for example [37]. However, equation (5) suggests a limit on how much the spontaneous emission rate $\gamma$ should be increased through the Purcell effect. The two-photon interference visibility reaches a maximum when $\gamma=\sqrt{\alpha r / 2}$. When $\gamma$ is increased further, the photon wavepackets become too short relative to the time uncertainty associated with the initial relaxation into the lowest-energy single-exciton state. For reasonable parameter values $1 / \alpha=1 \mathrm{~ns}$ and $1 / r=10 \mathrm{ps}$, this gives $\tau_{r}=1 /\left(2 \gamma_{\max }\right) \approx 70 \mathrm{ps}$. This is not much shorter than the shortest lifetimes already observed with the present devices. Therefore, the benefits of improved cavities will be lost unless the time uncertainty in the excitation process can be reduced. 
This time uncertainty is a result of the incoherent excitation scheme we have adopted. This scheme was chosen for its experimental simplicity. Eventually, however, one must consider coherent excitation schemes, such as vacuum-stimulated Raman processes [5, 38], that have been studied in the development of atomic single-photon sources. Such schemes might be possible with quantum dots as well [39].

\section{Acknowledgments}

This work was supported by MURI UCLA/0160-G-BC575 and 'Nano-Photonic and Electron Devices Technology', Focused Research and Development Project for the Realization of the World's Most Advanced IT Nation, MEXT. This work has been supported in part by the MURI Center for Photonic Quantum Information Systems (ARO/ARDA program DAAD19-03-1-0199). We thank A Scherer and T Yoshie from Caltech for providing access to CAIBE and for help with fabrication.

\section{References}

[1] Brunel C, Lounis B, Tamarat P and Orrit M 1999 Phys. Rev. Lett. 832722

[2] Lounis B and Moerner W E 2000 Nature 407491

[3] Kurtsiefer C, Mayer S, Zarda P and Weinfurter H 2000 Phys. Rev. Lett. 85290

[4] Beveratos A, Kuhn S, Brouri R, Gacoin T, Poizat J-P and Grangier P 2002 Eur. Phys. J. D 18191

[5] Kuhn A, Hennrich M and Rempe G 2002 Phys. Rev. Lett. 89067901

[6] Strauf S, Michler P, Klude M, Hommel D, Bacher G and Forchel A 2002 Phys. Rev. Lett. 89177403

[7] Michler P, Kiraz A, Becher C, Schoenfeld W V, Petroff P M, Zhang L, Hu E and Imamoglu A 2000 Science 2902282

[8] Santori C, Pelton M, Solomon G, Dale Y and Yamamoto Y 2001 Phys. Rev. Lett. 861502

[9] Zwiller V, Blom H, Jonsson P, Panev N, Jeppesen S, Tsegaye T, Goobar E, Pistol M-E, Samuelson L and Bjork G 2001 Appl. Phys. Lett. 782476

[10] Yuan Z, Kardynal B E, Stevenson R M, Shields A J, Lobo C J, Cooper K, Beattie N S, Ritchie D A and Pepper M 2002 Science 295102

[11] Vuckovic J, Fattal D, Santori C, Solomon G S and Yamamoto Y 2003 Appl. Phys. Lett. 823596

[12] Moreau E, Robert I, Gerard J-M, Abram I, Manin L and Thierry-Mieg V 2001 Appl. Phys. Lett. 792865

[13] Pelton M, Santori C, Vuckovic J, Zhang B, Solomon G S, Plant J and Yamamoto Y 2002 Phys. Rev. Lett. 89 233602

[14] Santori C, Fattal D, Vuckovic J, Solomon G S and Yamamoto Y 2002 Nature 419594

[15] Gisin N, Ribordy G, Tittel W and Zbinden H 2002 Rev. Mod. Phys. 74145

[16] Monroe C 2002 Nature 416238

[17] Knill E, LaFlamme R and Milburn G J 2001 Nature 40946

[18] Waks E, Santori C and Yamamoto Y 2002 Phys. Rev. A 66042315

[19] Knill E 2003 Preprint quant-ph/0312190

[20] Bimberg D, Grundmann M and Ledentsov N N 1999 Quantum Dot Heterostructures (Chichester: Wiley)

[21] Gerard J M, Sermage B, Gayral B, Legrand B, Costard E and Thierry-Mieg V 1998 Phys. Rev. Lett. 811110

[22] Solomon G S, Pelton M and Yamamoto Y 2001 Phys. Rev. Lett. 863903

[23] Vuckovic J, Pelton M, Scherer A and Yamamoto Y 2002 Phys. Rev. A 66023808

[24] Purcell E M 1946 Phys. Rev. 69681

[25] Landin L, Miller M S, Pistol M-E, Pryor C E and Samuelson L 1998 Science 280262

[26] Santori C, Fattal D, Vuckovic J, Solomon G S, Waks E and Yamamoto Y 2003 Preprint cond-mat/0308323

[27] Gayral B and Gerard J M 1999 J. Lightwave Technol. 172089 
[28] Santori C, Solomon G S, Pelton M and Yamamoto Y 2002 Phys. Rev. B 65073310

[29] Santori C, Fattal D, Pelton M, Solomon G S and Yamamoto Y 2002 Phys. Rev. B 66045308

[30] Moreau E, Robert I, Manin L, Thierry-Mieg V, Gerard J-M and Abram I 2001 Phys. Rev. Lett. 87183601

[31] Kamada H, Ando H, Temmyo J and Tamamura T 1998 Phys. Rev. B 5816243

[32] Kulakovskii V D, Bacher G, Weigand R, Kummell T, Forchel A, Borovitskaya E, Leonardi K and Hommel D 1999 Phys. Rev. Lett. 821780

[33] Hong C K, Ou Z Y and Mandel L 1987 Phys. Rev. Lett. 592044

[34] Fearn H and Loudon R 1989 J. Opt. Soc. Am. B 6917

[35] Bylander J, Robert-Philip I and Abram I 2003 Eur. Phys. J. D 22295

[36] Bayer M and Forchel A 2002 Phys. Rev. B 65041308

[37] Vuckovic J and Yamamoto Y 2003 Appl. Phys. Lett. 822374

[38] Hennrich M, Legero T, Kuhn A and Rempe G 2000 Phys. Rev. Lett. 854872

[39] Kiraz A, Atature M and Imamoglu A 2004 Preprint quant-ph/0308117 